\title{
Effects of carbon monoxide-releasing molecules on cGMP levels and soluble guanylate cyclase activity
} Antonia Marazioti ${ }^{1}$, Padmamalini Baskaran², Annie Beuve², Carlos Romao3, Anastasia Pyriochou*1 and Andreas Papapetropoulos ${ }^{1}$

\author{
Address: ${ }^{1}$ Lab of Molecular Pharmacology, Dept of Pharmacy, University of Patras, Greece, ${ }^{2}$ Dept of Pharmacology and Physiology, UMDNJ, New \\ Jersey, USA and ${ }^{3}$ Alfama, Inc, Lisbon, Portugal \\ Email: Anastasia Pyriochou* - anastassouli@yahoo.com \\ * Corresponding author
}

from 4th International Conference of cGMP Generators, Effectors and Therapeutic Implications

Regensburg, Germany. 19-21 June 2009

Published: II August 2009

BMC Pharmacology 2009, 9(Suppl I):P45 doi:I0.1186/147I-2210-9-SI-P45

This abstract is available from: http://www.biomedcentral.com//47I-22/0/9/SI/P45

(C) 2009 Marazioti et al; licensee BioMed Central Ltd.

\section{Background}

Carbon monoxide $(\mathrm{CO})$ is a signaling molecule that controls many physiological processes in mammalian tissues. CO gas activates sGC by 3 -4-fold and exerts vasodilatory effects through elevation of cGMP. Certain transition metal carbonyls liberate $\mathrm{CO}$ in a controlled fashion and function as CO-releasing molecules (CORMs). Some of these agents have a variety of pharmacological effects and have yielded encouraging results in preclinical models, where CORM administration alleviates inflammatory processes and cardiovascular disorders. The aim of the present work was to investigate whether the pharmacological effects of CORMs could be attributed to sGC activation. To this end, we selected 12 CORMs and tested their ability to alter cGMP levels.

\section{Materials and methods}

Rat aortic smooth muscle cells (RASMC) were incubated with IBMX ( $1 \mathrm{mM})$ for $5 \mathrm{~min}$. Cells were then treated with vehicle, sodium nitroprusside (SNP) $10 \mu \mathrm{I}, \mathrm{CO}$ and/or CORMs at various concentrations (1-100 $\mu \mathrm{I})$ for $15 \mathrm{~min}$; cGMP was extracted and measured by enzyme immunoassay. To establish co-cultures we seeded endothelial cells on confluent RASMCs; co-cultures were then treated a CORMs, in the presence or absence of bradykinin (10 $\mu \mathrm{I})$. Basal and DEA-NO $(1 \mu \mathrm{I})$-stimulated guanylyl cyclase activity was also measured in the presence of CORMs (10 and $100 \mu \mathrm{I})$.

\section{Results}

CO and 4 of the CORMs (ALF 153, 157, 436 and CORM2) increased cGMP levels in smooth muscle cells, while the rest of the compounds failed to alter cGMP production. The CORM-stimulated increase was minor (50$300 \%$ over basal) compared to the effects seen with the NO-donor, SNP (at least 100-fold). CORM-3, a widely used CORM, decreased basal cGMP levels in RASMC. Interestingly, $\mathrm{CO}$ and CORMs modestly reduced SNPstimulated cGMP formation in RASMC. The inactive forms of CORMs (compounds lacking the ability to liberate $\mathrm{CO}$ ) did not affect SNP-induced cGMP accumulation. CORMs also reduced bradykinin-stimulated cGMP levels in endothelial-smooth muscle co-cultures. In sGC activity assays using purified recombinant human sGC, CORMs were ineffective in stimulating cGMP production. However, CORMs blocked up to $80 \%$ of DEA-NO-induced sGC activity.

\section{Conclusion}

Collectively, our findings suggest that CORMs, due to their different chemical nature and physico-chemical properties may have complex and indirect effects on the NO/cGMP pathway, resulting in either stimulation or inhibition of intracellular cGMP levels. 